\title{
Repeated Inescapable Stress Produces a Neuroleptic-like Effect on the Conditioned Avoidance Response
}

\author{
Arnold J. Friedhoff, M.D., Kenneth D. Carr, Ph.D., Suzan Uysal, Ph.D.,
} and Jack Schweitzer, Ph.D.

This study tests the hypothesis that the dopaminergic system mediates a restitutive response by decreasing its own activity in the face of events like persistent inescapable stress that threaten to interrupt organized mental activity. It is well established that neuroleptic drugs inhibit the conditioned aroidance response (CAR). but not the escape response, probably via a reduction in subcortical dopaminergic activity. We trained rats to perform the CAR and then subjected them to acute and chronic stress to determine whether this would result in

KEY WORDS: Conditioned aroudance response: Dopamme; Stress; Restitutire systems

Neuroleptic drugs are typically screened for their antipsychotic potential by testing their ability to inhibit the conditioned avoidance response (CAR) without inhibiting the escape response (Arnt 1982; Davidson and Weidley 1976; Niemegeers et al. 1969). Inhibition of the CAR is believed to be mediated by a reduction in dopaminergic (DAergic) activity in both the striatum and nucleus accumbens (Koob et al. 1984). It has also been shown that stress produces an increase in the release of dopamine (DA) in the medial prefrontal cortex (Deutch et al. 1985; Thierry et al. 1976) and that this increase is believed to reduce DAergic activity in sub-

From Millhauser Laboratorie's, Department of P'suchiatry, Neu Yorh University School of Medicine, Ve'w York.

Address correspondence to Dr. Arnold I. Friedhotf, Millhauser Laboratories, Room HN-323, New York Linversity Medical Center. 550 First Avenue, New York, NY 100 It

Received October 3, 1994; revised Nosember 7 . 1494; accepted lanu. ary 15,1995 inhibition of the CAR. Rats subjected to twice daily tailshock stress for 8 days showed inhibition of the CAR and a reduction in dopamine (DA) utilization in the nucleus accumbens. These findings are compatible with the hypothesis that an endogenous DA-dependent mechanism exists that mimics neuroleptic effects in the face of repeated stress. In humans this response may serve as a protection against psychotic decompensation from chronic endogenous or exogenous insult. [Neuropsychopharmacology 13:129-138, 1995]

cortical DAergic terminal fields (Deutch et al. 1990; Louilot et al. 1989; Pycock et al. 1980). Thus stress, or perhaps repeated stress, by increasing DAergic activity in the $\mathrm{MPFC}$ and reducing DAergic activity in subcortical nuclei, might inhibit the CAR.

We previously pointed out that the DAergic syste'm, among its many functions, appears to act as a buffer or restitutive system (Friedhoff 1985, 1986, 1988). Neuroleptics, which reduce DAergic activity initially by blocking DA receptors and later by reducing DA synthesis and release (Bunney \& Grace 1978; Skirboll \& Bunney 1979) are effective against psychotic symptoms of diverse etiology. Thus reduction of DAergic activity suppresses psychotic symptoms of all types, rather than those of a particular mental disorder.

It is well known that neuroleptics, in the course of producing antipsychotic effects, often increase the flatness of affective responses. Flat affect may be secondary to the decreased DAergic activity produced by neuroleptics because Parkinsonism, a hypodopaminergic syndrome, has associated flat affect as a primary symptom. Reducing DAergic activity with neuroleptics has 
as its major action the attenuation of so-called positive symptoms, but neuroleptics also produce or intensify affectual flattening. Interestingly patients with negativesymptom schizophrenia also show flat affect as a primary symptom (Andreasen 1979, 1989; Andreasen et al. 1981; Andreasen and Olsen 1982).

We wanted to test the idea that there is an endogenous restitutive system that acts like a neuroleptic in the face of events that threaten to destabilize mental function. As mentioned, all neuroleptics inhibit the CAR. Thus we proposed that an inescapable threat to the integrity of mental function would produce inhibition of the CAR, just as a neuroleptic would. As a potential mental destabilizing situation, we chose repeated inescapable stress. A study was undertaken to determine if repeated inescapable stress could produce inhibition of the CAR. This would be evidence of a physiological response that could mimic that produced by neuroleptics. A further aim of this study was to determine whether repeated stress could produce adaptive changes in the brain DAergic system that are comparable to those induced by neuroleptics.

The study consisted of three experiments: In Experiment 1 , three stressors were evaluated to identify one, other than footshock, that strongly and preferentially activates the mesocortical DA pathway. The need to exclude footshock stems from the confounding effect that exposure to inescapable footshock could have on performance in the CAR paradigm where footshock is used as the unconditioned stimulus (see later). In Experiment 2 , brain regional DA utilization was evaluated immediately following a CAR session in order to identify brain regions in which DA is utilized when rats successfully execute the CAR. An index of DA utilization is achieved by measuring tissue levels of DA and its principle metabolites, dihydroxyphenylacetic acid (DOPAC) and homovanillic acid (HVA). A ratio of DOPAC or HVA to DA is calculated, and an increase or decrease in these ratios reflects an increase or de.crease, respectively, in DA metabolism and utilization. In Experiment 3, the effect of acute stress and chronic stress on CAR performance was determined in order to evaluate whether stress produces a "neuroleptic-like" inhibition of the CAR. Brain regional DA utilization was then evaluated in an effort to correlate changes in CAR performance with changes in DAergic activity in chronically stressed rats. The three stressors were mild in termittent tailshock, witnessing a conspecific receiving mild intermittent tailshock, and exposure to the chamber (i.e., residual olfactory stimuli) in which two rats had just received the preceding two stress treatments

\section{MATERIALS AND METHODS}

\section{Experiment 1}

Male Sprague-Dawley rats $(30(0-350 \mathrm{~g})$ were housed in the general animal facility with ad libitum access to food and water for a period of at least 7 days before use.

Treatments. Each of four treatment groups contained 8 rats (because of occasional sampling problems, replicate tissue samples were not available for assay in a few (ases). Each rat received a single application of the assigned treatment for a duration of 20 minutes. Following treatment, rats were returned to home cages for 5 minutes and then sacrificed by decapitation.

Tailshock Stress. Each rat was placed in a cylindrical plexiglas restraining tube for approximately 3 minutes so that tail electrodes could be affixed. Tail electrodes were constructed of a 1-cm length of rubber tubing with two stainless steel machine screws inserted so that the heads extended slightly into the lumen of the tubing. The tubing fit snugly at the base of the tail with the screw heads making contact on the left and right side. To ensure good electrical conduction, each screw head was first coated with Cambridge electrode jelly. Electrical stimulation was delivered by a Grass S44 stimulator in combination with a PSIU5 stimulus isolation unit from which wires were connected to the exterior length of each machine screw. For tailshock treatment, rats were placed on the floor of a 36- $\times 36-\times 36-\mathrm{cm}$ plexiglas chamber but were further contained within a $25-\times$ $15-\times 15-\mathrm{cm}$ wire mesh enclosure. BRS/LVE electromechanical equipment and a Lafayette interval timer were used to deliver a 0.2 -second duration tailshock every 30 seconds for a period of 20 minutes. Tailshock intensity w'as adjusted on the basis of behavioral criteria; specifically, tailshock intensity was increased until the rat displayed a clear orientation response to the base of the tail. Orientation was sometimes but not always accompanied by a brief vocalization.

Witness Stress. Each "witness" was placed within the plexiglas chamber but outside of the wire mesh enclosure (see above) for the duration of another rats' tailshock session.

Olfactory Stress. Immediately after removing a tailshock witness pair from the plexiglas chamber, a third rat was placed in the chamber and confined there for a period of 20 minutes. Following removal of this rat, the plexiglas chamber and wire mesh enclosure were washed thoroughly before introducing the next tailshock witness pair for treatment.

Control. Control rats were maintained in home cages and then sacrificed.

Preparation of Tissue and Biochemical Assay. Following decapitation, brains were rapidly extracted, blocked, and placed on a cryostat chuck on the $-40^{\circ} \mathrm{C}$ quickfreeze platform of an IEC Minotome. One-mm coronal sections were cut at $-10^{\circ} \mathrm{C}$, placed on microscope slides, and transferred to a bed of powdered dry ice for removal of brain regions. The medial prefrontal cor- 
tex was cut from two serial sections using a scalpel blade. The nucleus accumbens was punched from two serial sections using a 1-mm-diameter stainless steel needle. The claudate nucleus (head) was punched from two serial sections using a 1.5 -mm-diameter needle. The samples were immediately transferred to tubes on dry ice.

Analysis of Dopamine and Metabolites. Homogenates of tissues, prepared by sonication in $0.1 \mathrm{M}$ perchloric acid, were centrifuged and, to a portion of the supernatant was added 3,4-dihydroxybenzylamine as internal standard for DA and DOPAC assays and, to another portion, 3-hydroxy-4-methoxyphenylacetic acid (isoHVA) as internal standard for HVA analysis. Portions of these solutions, without further purification, were injected onto a Supelcosil C-18 DB column (Supelco). The mobile phase was prepared by mixing $12 \mathrm{~g}$ of monosodium phosphate, $160 \mathrm{mg}$ of disodium EDTA, $440 \mathrm{mg}$ of heptane sulfonic acid monohydrate, $60 \mathrm{ml}$ of methanol, and sufficient distilled water to make about $1.8 \mathrm{~L}$ of solution. The $\mathrm{pH}$ was adjusted to 3.0 with $85 \%$ phosphoric acid and then brought to $2 \mathrm{~L}$ with water. This was filtered and degassed. Taking $1 \mathrm{~L}$ of the solution, $70 \mathrm{ml}$ were removed and replaced with $70 \mathrm{ml}$ of methanol. The flow rate was $1 \mathrm{ml} / \mathrm{min}$ for DA and DOPAC and $1.7 \mathrm{ml} / \mathrm{min}$ for HVA. The Coulochem Model 5100A (ESA) electrochemical detector was set at $0.47,-0.28$, and 0.45 for detectors one and two and conditioning cell, respectively.

Data Analysis. For each rat, indices of brain regional DA utilization were obtained by calculating the ratios of DOPAC/DA and HVA:DA. Effects of stressors on the two different ratios were determined for each brain region using separate one-way analysis of variance (ANOVA), which, in the case of significant $F$ values (i.e., $p<.05$ ), were followed by Dunnett comparisons of each stress treatment mean with the control mean.

\section{Experiment 2}

While the CAR is a classical behavioral screen for DAergic antagonist antipsychotic drugs, the anatomy of DA utilization during CAR performance has not, to our knowledge, been investigated. From microinjection mapping studies, using DA antagonists and 6-OHDA, it appears that subcortical DAergic activity is essential to CAR acquisition while cortical DAergic activity is not (Ashford and Jones 1976; Koob et al. 1984; Sherman et al. 1982). In order to further explore the relationship between regional DA utilization and the CAR, regional DA utilization was evaluated in rats that were sacrificed immediately following a session of successful CAR performance.

Twenty male Sprazui-Dawiey rats (250-300 g) were trained to perform in a twi-lid active avoidance task using the fully automated Omnitech (Columbus, $\mathrm{OH}$ ) Shuttle Scan system interfaced with an IBMXT. A training session consisted of 30 discrete trials separated by 30 -second intertrial intervals. Each trial began with presentation of a 10 -second auditory CS pulsed at $2.5 \mathrm{~Hz}$. On trials where animals did not perform a shuttle response during the CS (avoidance), a 0.5-mA scrambled footshock was delivered via the stainless steel grid floor. Footshock continued until the animal performed a shuttle escape response or for a maximum of 20 seconds. The number of avoidance and escape responses and their corresponding latencies were automatically recorded as were the number of noncontingent shuttle responses emitted during intertrial intervals. Daily training sessions continued until animals displayed a criterion avoidance rate of at least $80 \%$. Following fulfillment of the behavioral criterion, trained rats remained in home cages for 14 days. On the fifteenth day, half of the rats performed in a 30-trial CAR session while half remained in home cages immediately prior to sacrifice. Tissue samples were obtained and analyzed as described in Experiment 1.

Data Analysis. Inasmuch as the goal of this experiment was to identify brain regions in which indices of DA utilization are elevated as a result of CAR performance, one-tailed $t$-tests were used to compare DOPAC/DA and HVA/DA ratios within each brain region of control and experimental groups.

\section{Experiment 3a}

In this experiment, rats were trained to behavioral criteria in the CAR paradigm, and the effects of tailshock and witness stress on CAR performance were determined. For each stressor, effects of acute and chronic exposure were compared. In light of the observation that chronic tailshock did inhibit CAR performance (see below), brain regional DA utilization, following a CAR test, was evaluated to determine whether CAR inhibition correlated with decreased DAergic activity.

Eighteen male Sprague-Dawley rats (250-300 g) were trained to the behavioral criterion in the CAR paradigm and then divided into three experimental groups. One group was subsequently exposed to tailshock stress treatments, one to witness stress treatments, and the third group served as control. On the first day of experimental testing, each rat was subjected to a single application of their assigned treatment. This was immediately followed by a CAR test, made to evaluate the effects of acute stress on CAR performance. From the second through ninth days, each rat received its assigned treatment twice daily (A.M. and P.M.). Throughout this 8-day period there was no CAR testing. Control rats were transported to and from the lab each day, receiving neither stress treatments nor CAR testing. On 
the tenth experimental day, 20 hours after the most recent stress treatment, all rats received a CAR test.

Data Analysis. CAR performance on acute and chronic stress days was expressed as the change from baseline and analyzed by two-way ANOVA (groups $x$ test day) with repeated measures on the factor of test day. A significant interaction effect was followed by post hoc Tukey comparisons between groups on particular test days. Following our observation that chronic tailshock, but not witness stress, decreased CAR performance when tested 20 hours after the last stress treatment, the effect of a stress treatment immediately prior to CAR testing was examined by giving these same rats additional CAR tests. Twenty-four hours after the initial post-chronic stress CAR test, rats received a CAR test immediately following exposure to their usual stress treatment. Twenty-four hours later, another CAR test was conducted. Thus, the CAR performance documented 20 hours post-chronic stress was compared with: (1) CAR performance 24 hours later when preceded by acute application of the same stress treatment, and (2) CAR performance 24 hours after (1). A repeated measures A.NOVA and post hoc Dunnett com. parisons were used to compare the second and third CAR performances with the first.

To analyze differences in DOPAC/DA and HVA DA ratios between treatment groups, one-way ANOVAs were run for each measure in each brain region. In cases where significant $F$ values were obtained $(p<.05)$, Dunnett comparisons were used to compare treatment means with the control mean. We carried out one-tailed
Dunnett comparisons because we predicted that DA utilization would decrease as a result of stress. In the chronic tailshock stress group, where decreased CAR performance and decreased DA utilization in nucleus accumbens were found, the relationship between these two events was evaluated by calculating a Pearson correlation coefficient.

\section{Experiment $3 b$}

The purpose of this experiment was to replicate the finding that chronic tailshock stress impairs CAR performance and decreases DA utilization in nucleus accumbens in a manner that correlates with CAR performance.

Twelve male Sprague-Dawley rats (250-300 g) were trained to behavioral criteria in the CAR paradigm. Six then received twice-daily tailshock stress for 8 consecutive days while the remaining 6 were only transported to and from the laboratory. No CAR testing occurred during this 8-day period. Twenty-hours following the last stress treatment, rats received a CAR test immediately followed by sacrifice and processing of brains for biochemical assay.

\section{RESULTS}

\section{Experiment 1}

The object of this experiment was to find a stressor that would activate the $\mathrm{MPFC}$ but would have no effects on striatum or nucleus accumbens before acquisition of the CAR.

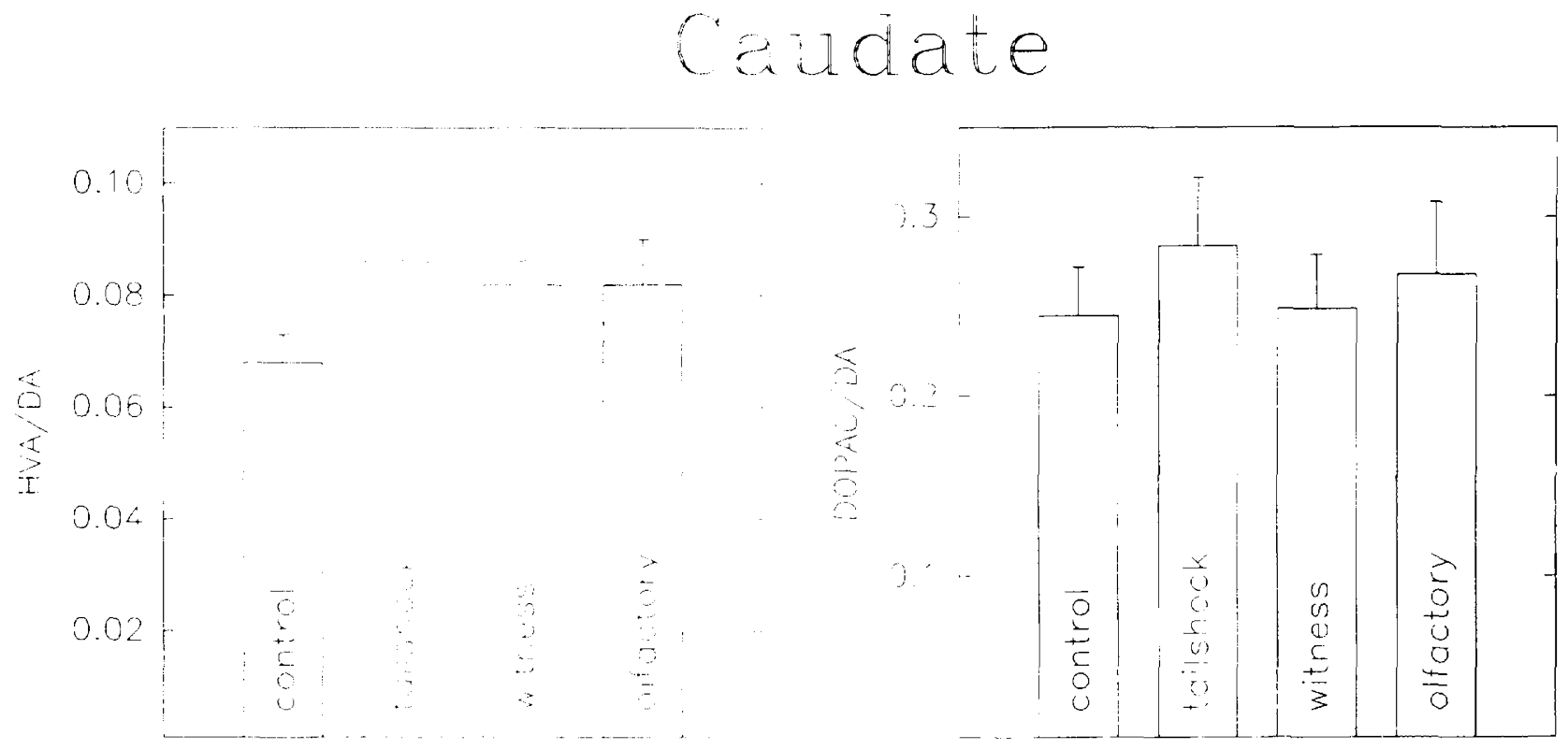

Figure 1. Following acute application of a stressor, rats were sacrificed and concentrations of DA and metabolites were measured in caudate nucleus. DA utilization expressed as HVA/DA and DOPAC/DA is displayed. A significant increase in either ratio, compared to control, would signify an increase in DA utilization. 
Table 1. Regional DA Utilization Following CAR Performance ${ }^{a}$

\begin{tabular}{|c|c|c|c|c|}
\hline & \multicolumn{2}{|c|}{ HVA $/ \mathbf{D A}^{b}$} & \multicolumn{2}{|c|}{$\mathbf{D O P A C} \mathbf{D A}^{b}$} \\
\hline & Control & CAR & Control & CAR \\
\hline Caudate & $0.868( \pm 0.008)$ & $0.1024( \pm 0.0006)$ & $0.1502( \pm 0.01)$ & $0.1485( \pm 0.01)$ \\
\hline Accumbens & $0.1253( \pm 0.016)$ & $0.1646( \pm 0.012)^{\imath}$ & $0.3019( \pm 0.026)$ & $0.3646( \pm 0.024)^{c}$ \\
\hline $\mathrm{mPFC}$ & $0.5396( \pm 0.105)$ & $0.5546( \pm 0.112)$ & $0.3625( \pm 0.029)$ & $0.5859( \pm 0.117)^{c}$ \\
\hline
\end{tabular}

\footnotetext{
"Rats were sacrificed immediately after performing the CAR, and it was found that DA utilization was increased in nucleus accumbens according to changes in HVA'DA and DOPAC/DA ratios and in the mPFC according to the DOPACIDA ratio.

${ }^{b}$ Mean \pm SEM

$p<.05$
}

In the caudate nucleus, neither DOPAC/DA $\left(F_{3,27}=\right.$ $0.43)$ nor HVA/DA $\left(F_{3,27}=2.16\right)$ were affected by stress treatments. In the nucleus accumbens, none of the stress treatments altered DOPAC/DA $\left(F_{3,26}=\right.$ $0.69)$, but HVA/DA was increased by tailshock stress $\left(F_{3,22}=3.57, p<.05 ;\right.$ Dunnett $\left.p<.05\right)$. In the medial prefrontal cortex, tailshock produced a significant increase in DOPAC/DA $\left(F_{3,24}=9.71, p<.001\right.$; Dunnett $p<.01)$, exceeding the control value by more than $100 \%$. HVA/DA in medial prefrontal cortex was similarly increased by tailshock $\left(F_{3,24}=7.03, p<.001\right.$; Dunnett $p<$ .01 ), again exceeding the control value by more than $100 \%$. HVA/DA values in medial prefrontal cortex were also significantly increased by witness stress (Dunnett, $p<.05$ ). Olfactory stress had no effect on DA utilization in any of the brain regions analyzed. These results are displayed in Figures 1 through 3.

\section{Experiment 2}

The object of this experiment was to determine whether an increase in DAergic activity in subcortical nuclei occurred in rats that had performed the CAR.

On the day of sacrifice, all CAR rats, except one, exceeded the $80 \%$ avoidance criterion. Indices of DA utilization in brains of these rats were therefore compared with those of controls. In the caudate nucleus, there was no difference between groups in DOPAC/DA or HVA/DA. In the nucleus accumbens, both DOPAC/ DA $[t(17)=1.75$, one-tailed $p<.05]$ and HVA/DA $[t(17)=1.88$, one-tailed $p<.05]$ was greater in CAR performers than in controls. CAR performers also displayed a significant increase in medial prefrontal DOPAC/DA $[t(16)=2.05, p<.05]$; (see Table 1 ).

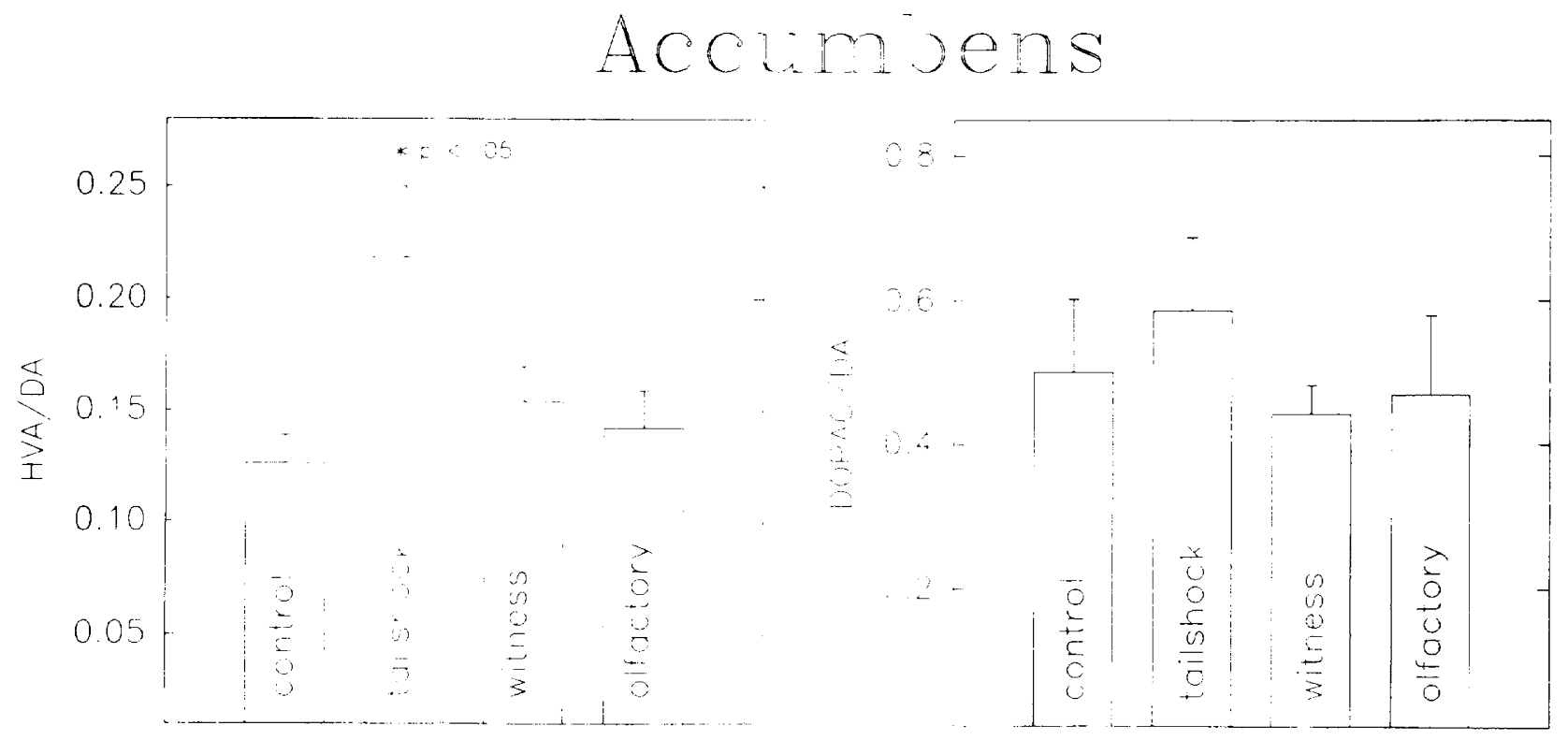

Figure 2. Following acute application of a stressor, rats were sacrificed and concentrations of DA and metabolites were measured in nucleus accumbens. DA utilization expressed as HVA/DA and DOPAC/DA is displayed. Tailshock stress significantly increased DA utilization as indexed by the HVA/DA ratio. 


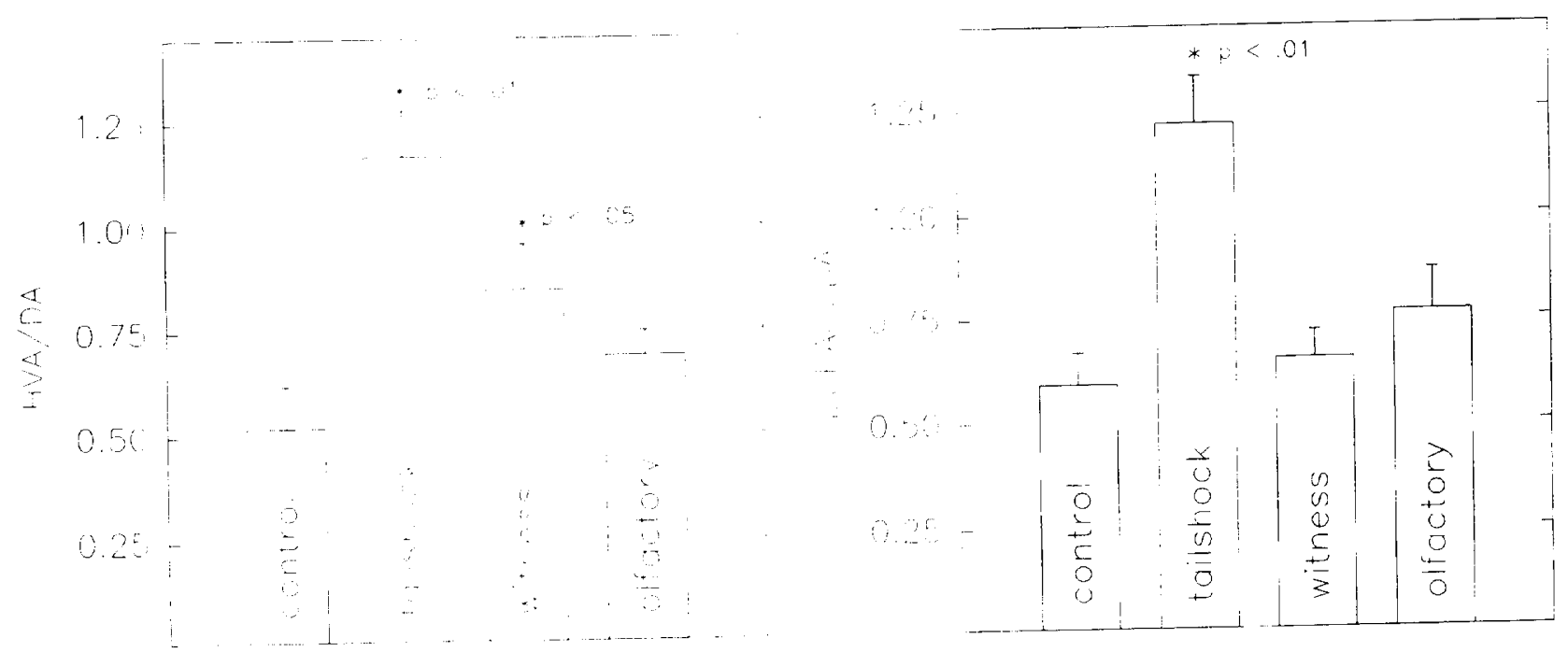

Figure 3. Following acute application of a stressor, rats were sacrificed and concentrations of DA and metabolites were measured in medial prefrontal cortex (mPFC). DA utilization expressed as HVA/DA and DOPAC/DA is displayed. DA utilization was increased by tailshock according to both measures and increased by witness stress according to the HVA/DA measure.

\section{Experiment 3}

Prior to the introduction of stress treatments, all rats reliably displayed minimum CAR performance of $80 \%$ ( 24 avoidance responses in a 30 -trial session). The mean

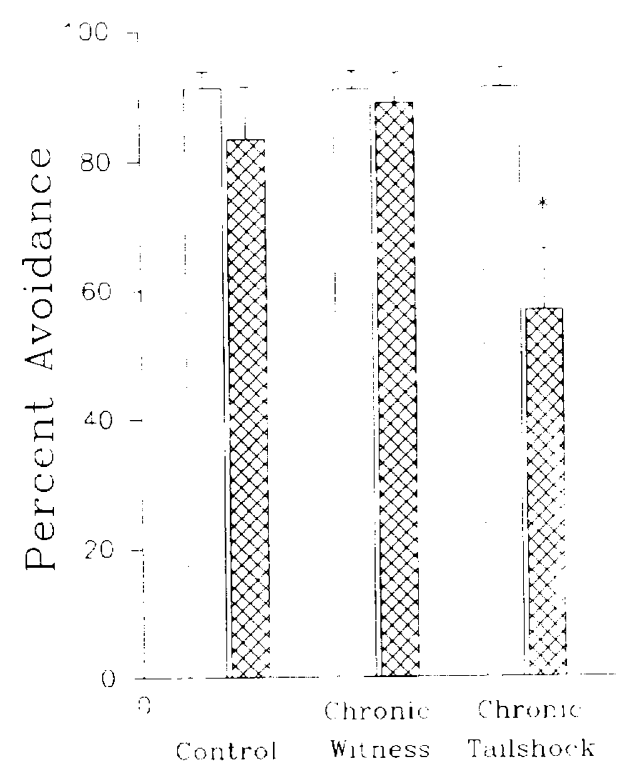

Figure 4. Three groups of rats were trained to behavioral criterion ( $>80 \%$ avoidance) and then subject to 8 days of twice daily tailshock stress, witnessing of tailshock stress, or control treatment. 24 hours later CAR performance was retested. For each treatment group, mean $( \pm$ SEM) percent avoidance prior to stress (clear bars) and following chronic stress (hatched bars) are displayed. Chronic tailshock stress decreased CAR performance $(p<.05)$.
CAR performance for each of the three experimental groups was virtually identical at $91 \%$ to $92 \%$. When the CAR was tested immediately following the first acute exposure to stress, all three groups continued to perform at the baseline level (i.e., $>90 \%$ ). Twenty hours after 8 days of twice-daily stress treatment, CAR performance of the tailshock stress group decreased significantly by comparison with the control group

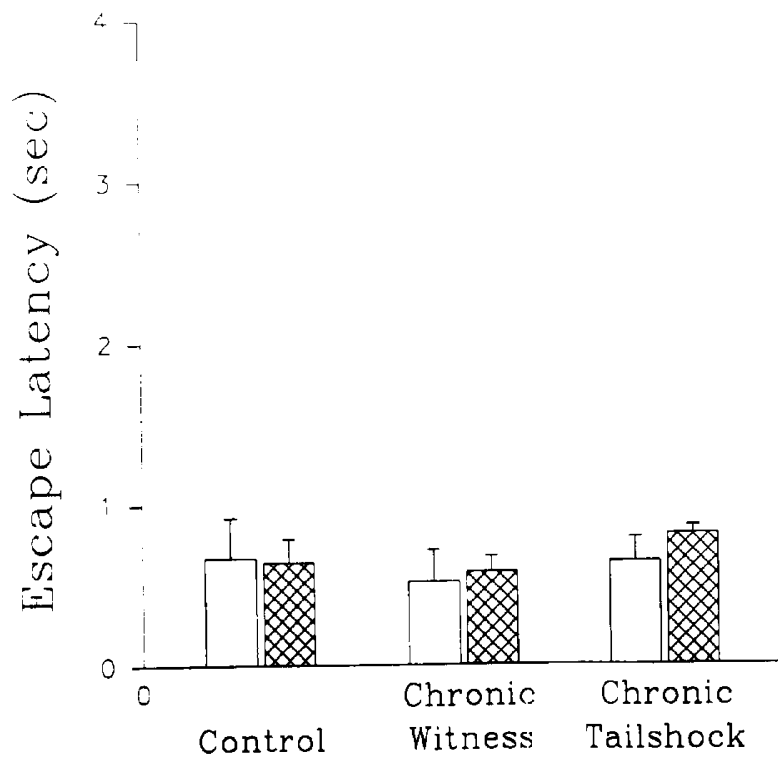

Figure 5. For each treatment group, escape latency, on trials where avoidance did not occur, prior to chronic stress treatment (clear bars) and following chronic stress (hatched bars), are displayed. No significant difference was found. 
Table 2. Percent Avoidance

\begin{tabular}{lccc}
\hline \multicolumn{3}{c}{ Post-Chronic Stress } \\
Rat & 24 Hours & $\begin{array}{c}\text { 48 Hours } \\
\text { (Preceded by Acute Stress) }\end{array}$ & 72 Hours \\
\hline 1 & 46.7 & 100.0 & 53.3 \\
2 & 53.3 & 80.0 & 66.7 \\
3 & 70.0 & 96.7 & 83.3 \\
4 & 30.0 & 43.3 & 83.3 \\
5 & 43.3 & 100.0 & 66.7 \\
$\bar{X} \pm$ SEM & $48.6 \pm 13.1$ & $84.0 \pm 21.7$ & $70.7 \pm 11.4$ \\
\hline
\end{tabular}

"CAR was tested at 24,48 , and 72 hours post chronic stress. The significant deficit seen at 24 hours was eliminated by pretreatment with an acute stress, 48 hours later. At 72 hours with no acute stress pretreatment, CAR performance was inhibited and no different from 24 hours post chronic stress (see test for $p$ values).

( $F_{\text {grrups }} \times$ days $=4.01, p<.05$; Tukey control vs. tailshock, chronic, $q=3.8, p<.05$; see Figure 4 ). Inspection of results for individual animals revealed that 5 of the 6 rats displayed a marked decrease in avoidance. The decreased performance in these rats was selective for avoidance behavior inasmuch as latency to escape footshock on trials where avoidance did not occur was short and no different from normal escape latency (Figure 5). Twenty-four hours later, when all rats received another CAR test immediately following an application of their assigned stress treatment, the witness stress rats continued to avoid at baseline levels. Interestingly, the tailshock stress rats, whose avoidance was inhibited

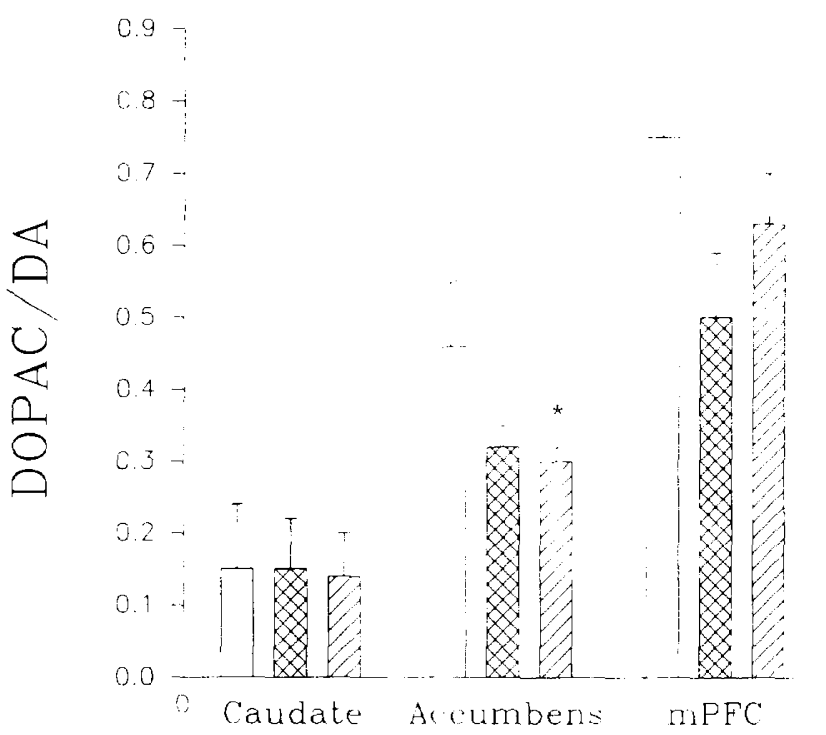

Figure 6. Following the final CAR test all rats were sacrificed and concentrations of DA and metabolites were measured in caudate nucleus, nucleus accumbens, and medial prefrontal cortex (mPFC). DA utilization expressed as DOPAC/DA is displayed for control (clear bars), chronic witness (hatched bars), and chronic tailshock (diagonal bars) groups. DOPAC/DA in the nucleus accumbens of chronic tailshock group was lower than in the control group $\left({ }^{*} p<.05\right)$ the day before, displayed a significantly improved CAR performance $\left(F_{2,10}=4.8, p<.05\right.$; Dunnett $p<.01$; see Table 2). After another 24 hours, most of these rats again displayed diminished CAR performance. CAR performance at this time was not significantly better than it was 24 hours after chronic stress.

Tissues taken immediately after the final CAR test revealed no differences between DOPAC/DA or HVA/ DA within the caudate nucleus or medial prefrontal cortex of the three different treatment groups. In the nucleus accumbens, however, only the tailshock stress group displayed a reduction in DOPAC/DA $\left[F_{2,13}=\right.$ $2.7, p=.11$, Dunnett tailshock $=p<.05]$. Both the witness stress and tailshock stress groups displayed reductions in $\mathrm{HVA} / \mathrm{DA} \mid F_{2,11}=5.83, p<.02$; Dunnett $_{\text {tailshock }}=$

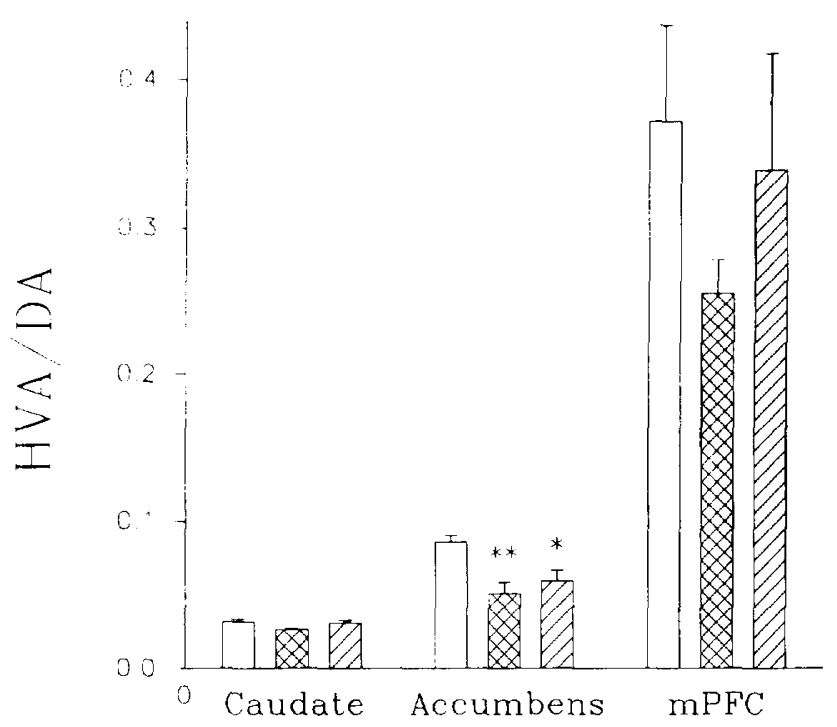

Figure 7. DA utilization expressed as HVA/DA is displayed for control (clear bars), chronic witness (hatched bars), and chronic tailshock (diagonal bars) groups. HVA/DA in the nucleus accumbens of both stress treatment groups was lower than in the control group $\left({ }^{*} p<.05,{ }^{* *} p<.01\right)$. 
$p<.05$; Dunnett witness $=p<.01 \mid$. These results are displayed in Figures 6 and 7.

In summary, only the tailshock stress group displaved inhibition of the CAR while both the witness and tailshock stress groups displayed a decrease in nucleus accumbens DA utilization following the CAR session. For rats in the tailshock group, there was a Pearson correlation between percent avoidance and HVA/DA of $r=+0.71$, although this correlation did not achieve statistical significance.

In experiment $3 \mathrm{~b}$ rats treated with chronic tailshock again displayed inhibition of the CAR (matched pairs $t(5)=6.17, p<.01$; Figure 8 ). In this experiment, unlike in experiment $3 a$, there was also a slight lengthening of the escape latency $[t(5)=2.54, p<.05$; Figure 9]. Indices of DA utilization were again reduced in nucleus accumbens of the chronic stress group, but did not reach significance, in contrast to the first experiment.

While conducting Experiment $3 b$ two additional noteworthy findings were observed. First, when rats received chronic tailshock treatment without being restrained several minutes before each treatment to affix the tail electrode, a significant C AR inhibition was not produced.

Second, chronic brief restraint, exactly as utilized for affixing tail electrodes, without tailshock also failed to affect CAR performance. Thus, it seems that the compound stress of brief restraint followed by tailshock, as used in Experiments 1, 3a, and 3b, may be necessary to induce inhibition of the CAR.

$.00-$

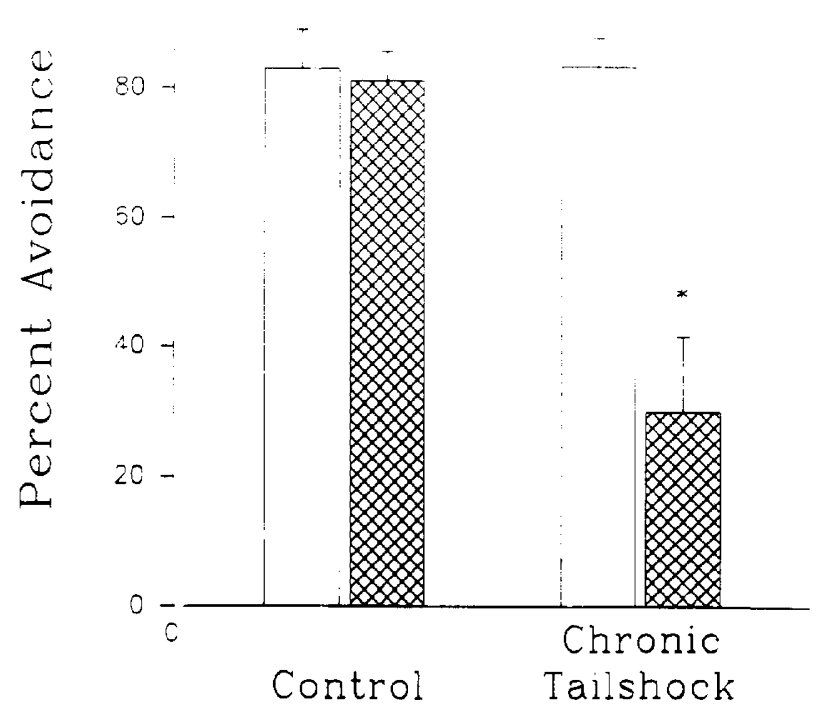

Figure 8. Two groups of rats were trained to behavioral criteria in the CAR ( $>80 \%$ avoidance), subject to twice daily tailshock stress or control treatment for 8 days, and 24 hours later retested in the CAR. Percent avoidance prior to (clear bars) and following (hatched bars) chronic stress treatment are displayed $\left({ }^{*} p<.01\right)$.

\section{DISCUSSION}

It is clear from the results of Experiment 1 that tailshock stress produced a large increase in cortical DA utilization, and to a lesser extent in nucleus accumbens. Witness stress was anatomically selective in stimulating DA utilization in medial prefrontal cortex; however, the effect was only manifest in the HVA/DA measure. The tailshock stress, therefore, seemed most suitable for purposes of subsequent experiments; however both tailshock and witness stress were used in Experiment 3 where effects of acute and chronic exposure on CAR performance were determined.

From the results of experiment 2 it appears that CAR performance entails DA utilization in nucleus accumbens and medial prefrontal cortex. The role of the nucleus accumbens is further supported by results of a recent microdialysis study in which leverpress avoidance was associated with increased nucleus accumbens DA utilization and performance was impaired by local injection of 6-OHDA (McCullough et al. 1993). Whether DAergic activity in nucleus accumbens is necessary for CAR performance has yet to be tested in our paradigm. In a one-way avoidance paradigm, Koob and coworkers (1984) found that DA blockade in nucleus accumbens alone is insufficient to inhibit acquisition of avoidance. They found that DA activity in nucleus accumbens and caudate nucleus had to be blocked simultaneously in order to inhibit avoidance. Two-way avoidance is a more complex task than one-way avoidance and may be dependent more on the functions that nucleus accumbens DA activity is proposed to mediate, including selective attention (Solomon and Staton 1982), re-

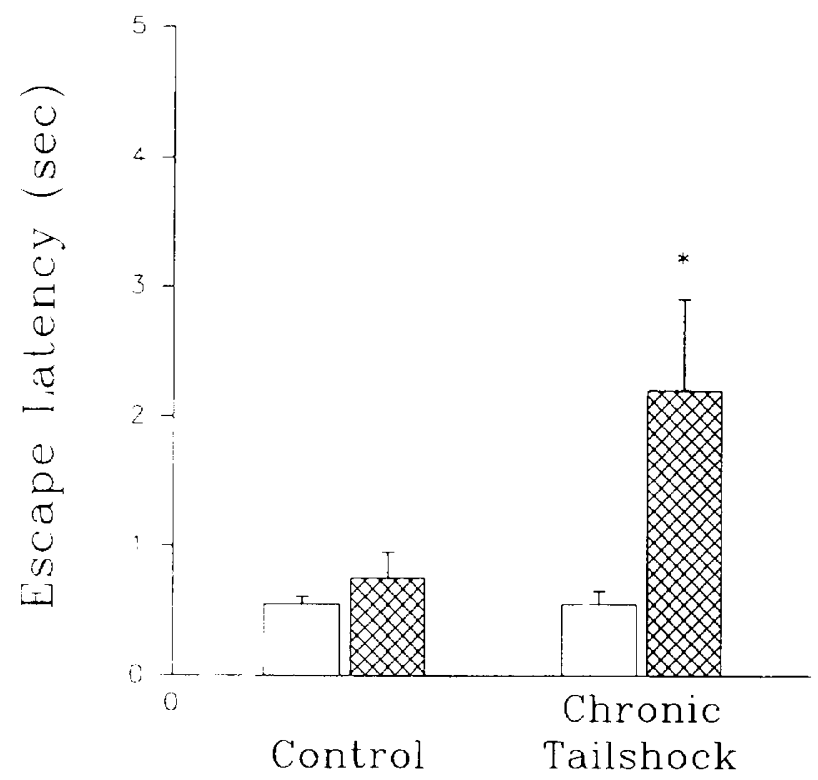

Figure 9. Mean (SEM) escape latency, on those trials where rats failed to avoid, prior to (clear bars) and following (hatched lurs) (hronic stress treatment are displayed $\left({ }^{*} p<.05\right)$. 
sponsiveness to environmental cues (van den Bos et al. 1991), and switching from ongoing behavior to another behavior (Taghzouti et al. 1985). Thus, whereas the first aim of experiment 3 (also see later) was to determine whether acute or chronic stress inhibits the CAR, an associated aim was to determine whether any observed inhibition of the CAR is accompanied by reduced DA utilization, particularly in the nucleus accumbens.

From the results of Experiment 3 it appears that chronic exposure to stressors that activate the mesocortical DA pathway reduce DA utilization in the nucleus accumbens during CAR performance and, in the case of tailshock stress, produce a marked impairment of CAR performance. From the findings of experiment 2 , which reveal an association between nucleus accumbens DAergic activity and CAR performance, along with the present association between CAR inhibition and reduced nucleus accumbens DA utilization in the tailshock group, a causal relationship between chronic stress, dampening of nucleus accumbens DAergic activity during the CAR, and CAR inhibition would seem possible; however, the finding that chronic witness stress reduces nucleus accumbens DA activity during the CAR, without inhibition of the CAR, refutes a simple causal connection. It might be hypothesized that reduced DA utilization in nucleus accumbens is necessary but not sufficient to cause inhibition of the CAR. This hypothesis would be compatible with the conclusion of Koob and coworkers (1984) that a 6-OHDA lesion of nucleus accumbens is necessary but not sufficient to block a one-wav CAR

The behavioral or physiological mechanism(s) underlying inhibition of CAR performance by chronic stress are not clear. The fact that chronic but not acute tailshock blocked the CAR would seem to rule out a "motor activation deficit." Weiss and coworkers (1975) have found that acute exposure to severe stress blocks instrumental escape and avoidance performance and depletes brain norepinephrine. Chronic exposure to such stress results in both noradrenergic and behavioral adaptation such that instrumental escape is restored. Thus, changes in instrumental behavior go in the direction opposite to those observed in the present study. A learning of "helplessness" would also seem unlikely to explain the CAR inhibition inasmuch as instrumental escape was not affected by the chronic stress treatment. One would expect "helplessness" to extend to all aversively motivated instrumental shuttle responses. Moreover, the transient restoration of CAR performance produced by an immediately preceding stress treatment would seem incompatible with learned helplessness, which should, if anything, be strengthened by an additional stress treatment prior to CAR testing (Seligman 1972). Although it is of general importance to understand the behavioral mechanism of the present CAR inhibition, the more important ques- tion for the present study is whether the physiological and/or behavioral mechanism of inhibition of the CAR is related to that produced by neuroleptic drugs.

It should be noted that even in the case of neuroleptic drugs, the behavioral mechanism of CAR inhibition is not clearly understood. For example, there are data to support proposals that the inhibition of the CAR results from the impairment of voluntary response initiation (Koob et al. 1984), the enhancement of fearinduced freezing behavior (Blackburn and Phillips 1990), or the blockade of incentive motivation (Beninger 1983). Replication of the chronic stress-induced inhibition of the CAR in Experiment 3b strengthens the hypothesis that exposure to chronic stress produces CAR inhibition similar to that produced by neuroleptic antipsychotic drugs. We also found, however, that an acute stressor temporarily restored CAR performance, for reasons that are not presently clear. It should be noted however that an additional 24 hours after the acute stressor, inhibition of the CAR produced by chronic stress was again evident. Thus, the enduring effect of chronic stress appears to be the inhibition of CAR performance. Inasmuch as there was a tendency for HVA/DA and DOPAC/DA in the nucleus accumbens to be reduced in the rats that displayed inhibition of the CAR, we cannot rule out the possibility that a modest decrease in accumbens DA utilization may be sufficient to affect the CAR when combined with other CNS changes not identified in this study. A continuing effort to elucidate the relationship between brain DAergic activity and CAR inhibition following chronic stress might benefit from use of microdialysis technology that allows monitoring of dynamic changes in DA activity online, and from investigation of additional subcortical DA terminal areas such as amygdala (Ashford and Jones 1976; Sherman et al. 1982).

Considering all of the data from these several experiments, it can be concluded that repeated tailshock stress, coupled with brief restraint, produces marked inhibition of the CAR. We are not proposing that neuroleptics or chronic stress produce a protective effect on mental function by inhibiting the CAR. Rather, inasmuch as all neuroleptics can produce inhibition of the CAR, this ability appears to be a marker of a dopaminedependent protective system. Thus these findings provide support for our earlier hypothesis that one physiological function of the DAergic system is to buffer stress and thereby prevent disorganization of mental function when the stress is chronic and inescapable. Stress, however, is believed, by some, to produce relapse in schizophrenia (Deutch 1993; Norman and Malla 1993). One could speculate that patients who succumb to stress have a defect in regulation of a DA-dependent protective system and cannot make the necessary downward adjustments without the assistance of neuroleptics. Defective function of this system could lead to vulnerability to psychosis. 


\section{ACKNOWLEDGMENTS}

This research was supported by NIMH Research Scientist Award MH14024 and Program Project Grant MH08618 both to Arnold J. Friedhoff. The authors are indebted to Mary Armour for outstanding technical assistance.

\section{REFERENCES}

Andreasen NC (1979): Affective flattening and the criteria for schizophrenia. Am J Psychiatry 136:944-947.

Andreasen NC (1989): The scale for assessment of negative symptoms (SANS): Conceptual and theoretical foundations. Brit J Psychiatry 155:49-52

Andreasen NC, Alpert M, Martz MJ (1981): Acoustic analy. sis: An objective measure of affective flattening. Arch Gen Psychiatry 38:281-285

Andreasen NC, Olsen S (1982): Negative vs. positive schizo. phrenia. Arch Gen Psychiatry 39:789-794

Arnt J (1982): Pharmacological specificity of conditioned avoidance response inhibition in rats: Inhibition by neuroleptics and correlation to DA receptor blockade. Acta Pharmacol Toxicol 51:321-329

Ashford J, Jones BJ (1976): The effects of intra-amygdaloid injections of 6-hydroxydopamine on avoidance responding in rats. Br J Pharmacol 56:255-261

Beninger RJ (1983): The role of dopamine in locomotor acticity and learning. Brain Res Rev 6:173-196

Bunney BS, Grace AA (1978): Acute and chronic haloperidol treatment comparison of effects on nigral dopaminergic cell activity. Life Sci 23:1715-1728

Blackburn JR, Phillips AG (1990): Enhancement of freezing behaviour by metoclopramide: Implications for neuroleptic-induced avoidance deficits. Pharmacol Biochem Behav 35:685-691

Davidson AB, Weidley E (1976): Differential effects of neu roleptic and other psychotropic agents on acquisition of avoidance in rats. Life Sci 18:1270-1284

Deutch AY (1993): Prefrontal cortical DA systems and the elaboration of functional corticostriatal circuits: Implications for schizophrenia and Parkinsons disease. Neural Trans 91:197-221

Deutch AY, Clark WA, Roth RH (1990): Prefrontal cortical dopamine depletion enhances the responsiveness of mesolimbic dopamine neurons to stress. Brain Res 521:311-315

Deutch AY, Tam SY, Roth RH (1985): Footshock and conditioned fear increase 3,4-dihydroxyphenylacetic acid (DOPAC) in the ventral tegmental area but not substantia nigra. Brain Res 333:143-146

Friedhoff AJ (1985): Restitutive processes in the regulation of behavior. In Alpert $\mathrm{M}$ (ed), Controversies in Schizophrenia: Change and Constancies, New York Guilford Press, pp 137-144

Friedhoff AJ (1986): A dopamine-dependent restitutive sy ;- tem for the maintenance of mental normalcy. In Burrell CD, Strand FL (eds.), Second Colloquium in Biological Sciences. New York, Ann NY Acad Sci 463:860-864

Friedhoff AJ (1988): Dopamine as a mediator of a central stabilizing system. Comments on the current status of the dopamine hypothesis of schizophrenia. Neuropsychopharmacology 1:189-191

Koob G, Simon H, Herman JP, Le Moal M (1984): Neurolepticlike disruption of the conditioned avoidance response requires destruction of both the mesolimbic and nigrostriatal dopamine systems. Brain Res 303:319-329

Louilot A, Le Moal M, Simon H (1989): Opposite influences of dopaminergic pathways to the prefrontal cortex or the septum on the dopaminergic transmission in the nucleus accumbens. An in vivo voltammetric study. Neurosci $29: 45-56$

McCullough LD, Sokolowski JD, Salamone JD (1993): A neurochemical and behavioral investigation of the involvement of nucleus accumbens dopamine in instrumental avoidance. Neurosci 52:919-925

Niemegeers CJE, Verbruggen FJ, Janssen PAJ (1969): The influence of various neuroleptic drugs on shock avoidance responding in rats. Psychopharmacol 16:161-174

Norman RW, Malla AK (1993): Stressful life events in schizophrenia, concepts and methodology. Brit J Psychiat 162:166-174

Pycock CJ, Carter CJ, Kerwin RW (1980): Effect of hydroxydopamine lesions of the medial prefrontal cortex on neurotransmitter systems in subcortical sites in the rat. J Neurochem 34:91-99

Seligman MEP (1972): Learned Helplessness. Ann Rev Med 23:407-412

Sherman AD, Petty F, Sacquitne JL (1982): Regional aspects of the delay of acquisition conditioned avoidance responding by chlorpromazine. Life Sci 30:1811-1815

Skirboll LR, Bunney BS (1979): The effects of acute and chronic haloperidol treatment on spontaneously firing neurons in the caudate nucleus of the rat. Life Sci 25:1419-1434

Solomon PR, Staton DM (1982): Differential effects of microinjections of D-amphetamine into the nucleus accumbens or the caudate putamen on the rat's ability to ignore an irrelevant stimulus. Bio Psychiatry 17:743-765

Taghzouti K, Simon H, Louilot A, Herman JP, Le Moal M (1985): Behavioral study after local injection of 6-hydroxydopamine into the nucleus accumbens in the rat. Brain Res 344:9-20

Thierry AM, Tassin JP, Blanc G, Glowinski J (1976): Selective activation of the mesocortical DA system by stress. Nature 263:242-244

van den Bos R, Ortiz GAC, Bergmans AC, Cools AR (1991): Evidence that dopamine in the nucleus accumbens is involved in the ability of rats to switch to cue-directed behaviours. Behav Brain Res 42:107-114

Weiss JM, Glazer HI, Pohorecky LA, Brick J, Miller NE (1975): Effects of chronic exposure to stressors on avoidanceescape behavior and on brain norepinephrine. Psychosom Med 37:522-534 